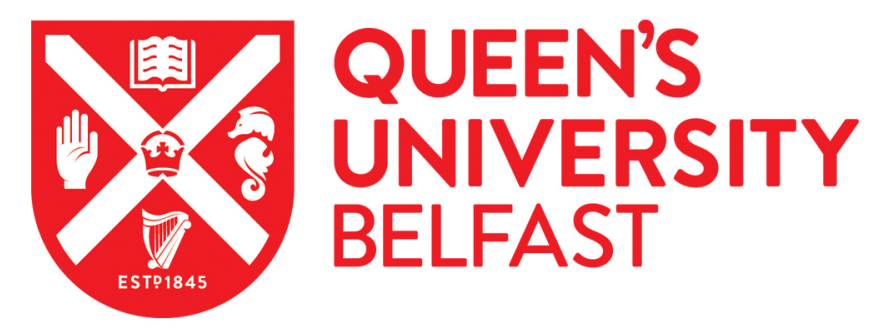

\title{
Drive-by bridge inspection from three different approaches
}

Kim, C. W., Isemoto, R., McGetrick, P. J., Kawatani, M., \& OBrien, E. J. (2014). Drive-by bridge inspection from three different approaches. Smart Structures and Systems, 13(5), 775-796.

https://doi.org/10.12989/sss.2014.13.5.775

\section{Published in:}

Smart Structures and Systems

\section{Document Version:}

Peer reviewed version

Queen's University Belfast - Research Portal:

Link to publication record in Queen's University Belfast Research Portal

Publisher rights

(C) 2014 KISTI Korean Institute of Science and Technology Information

\section{General rights}

Copyright for the publications made accessible via the Queen's University Belfast Research Portal is retained by the author(s) and / or other copyright owners and it is a condition of accessing these publications that users recognise and abide by the legal requirements associated with these rights.

Take down policy

The Research Portal is Queen's institutional repository that provides access to Queen's research output. Every effort has been made to ensure that content in the Research Portal does not infringe any person's rights, or applicable UK laws. If you discover content in the Research Portal that you believe breaches copyright or violates any law, please contact openaccess@qub.ac.uk. 


\title{
Drive-by bridge inspection from three different approaches
}

\author{
C.W. Kim ${ }^{1}$, R. Isemoto ${ }^{1}$, P.J. McGetrick ${ }^{1}$, M. Kawatani ${ }^{2}$, and E.J. OBrien ${ }^{3}$ \\ ${ }^{1}$ Department of Civil and Earth Resources Engineering, Kyoto University, Kyoto 615-8540, Japan \\ ${ }^{2}$ Department of Civil Engineering, Kobe University, Kobe, Japan \\ ${ }^{3}$ School of Civil, Structural \& Environmental Engineering, University College Dublin, Newstead, Belfield, Dublin \\ 4, Ireland
}

\begin{abstract}
This study presents a vibration-based health monitoring strategy for short span bridges utilizing an inspection vehicle. How to screen the health condition of short span bridges in terms of a drive-by bridge inspection is described. Feasibility of the drive-by bridge inspection is investigated through a scaled laboratory moving vehicle experiment. The feasibility of using an instrumented vehicle to detect the natural frequency and changes in structural damping of a model bridge was observed. Observations also demonstrated the possibility of diagnosis of bridges by comparing patterns of identified bridge dynamic parameters through periodical monitoring. It was confirmed that the moving vehicle method identifies the damage location and severity well.
\end{abstract}

Keywords: bridge engineering, bridge frequency, vehicle-bridge interaction (VBI), vibration-based health monitoring.

\section{Introduction}

Large portions of bridges located in municipalities are short span bridges, but have not been maintained properly because of budget restrictions of local governments. In Japan, for example, more than 85 per cent of bridges are classified as short span bridges with span length between $15 \mathrm{~m}$ and $50 \mathrm{~m}$. Developing a rapid and cost-effective tool for bridge health monitoring (BHM) focusing on short span bridges, therefore, is an important technical issue.

BHM at a global level using dynamic system parameters has been one of the most important approaches, and also has been intensively studied (e.g., Rizos et al. 1990, Doebling et al. 1996, Shifrin and Ruotolo 1999, Adeli and Jiang 2006, Siringoringo and Fujino 2006, Ni et al. 2008). The basic idea behind BHM using the dynamic system parameters is that frequency and damping characteristics, as well as mode 
shapes may provide useful information for the current health condition of bridges. The fundamental concept of this technology is that modal parameters are functions of a structure's physical properties. Therefore, a change in physical properties, such as reduced stiffness resulting from damage, will cause a change in these measurable modal properties (e.g. Wang and Fang 1986, Friswell and Mottershead 1994). Of course, applying sensors around expected or suspected damage substructures is one of the best approaches to detect damage. This is only effective, however, if the bridge structure has well defined damage models. For real bridge structures it is difficult to define a damage model differently from other structures such as automobiles, aerial vehicles, etc. Therefore, most precedent studies focusing on bridge health monitoring have specifically examined the global change of modal properties and quantities of bridge structures.

How to excite short span bridges is another challenge for vibration-based BHM because short span bridges are insensitive or sometimes impassive to external dynamic sources such as wind loads, ground vibrations, etc. Of course, normal traffic excitations are important dynamic sources, but a cautious approach is required to use traffic-induced vibrations of short span bridges because the traffic-induced vibration is a kind of non-stationary process (Kim et al. 2005).

Despite the non-stationary property of traffic-induced vibrations of bridges, the traffic excitation is an attractive dynamic source for the vibration-based health monitoring of short span bridges. The idea to utilize traffic-induced vibrations for health monitoring of short span bridges forms the basis of the drive-by bridge inspection using an inspection vehicle. A strong point of the drive-by bridge inspection is the ready excitement by the inspection vehicle. Another advantage is its rapidity, since the inspection vehicle acquires and processes vibration data of bridges while traveling on bridges. Theoretically the moving vehicle on bridges also carries components of bridge vibrations (e.g. Yang et al. 2004, Kim et al. 2005). Therefore three major functions can be expected from the inspection vehicle, such as an actuator, data acquisition and message carrier.

The U.S. Federal Highway Administration (FHWA) develop the High-speed Electromagnetic Roadway Mapping and Evaluation System (HERMES) as a drive-by inspection system focusing on the use of imaging radar for scanning bridge decks (FHWA 2001). Furukawa et al. (2007) employ vehicle's acceleration responses for pavement diagnostic. The feasibility of extracting bridge dynamic parameters such as natural frequency from the dynamic response of an instrumented vehicle has been verified theoretically (Yang et al 2004, Yang and Lin 2005, McGetrick et al. 2009, Gonzalez et al. 2010). Yang et al. (2004) find that the magnitude of the peak response 
in the vehicle acceleration spectra increased with vehicle speed but decreases with increasing bridge damping ratio. In a study by McGetrick et al. (2009) the bridge frequency and changes in bridge damping are extracted from the vehicle response but they find that it is difficult to detect both parameters in the presence of a rough road profile. Also, frequency matching between the vehicle and the bridge is highlighted by both Yang et al. (2004) and González et al. (2010) as being beneficial for frequency detection. Yang and Chang (2009) also carry out a parametric study which indicates some of the best conditions for frequency detection. Yin and Tang (2011) investigate the feasibility of detecting cable tension loss and deck damage of a cable-stayed bridge utilizing the vertical vibration of a vehicle analytically.

Adopting the inspection vehicle both as an actuator and for data acquisition is another way of carrying out the drive-by bridge inspection, which needs to implement wireless sensors on the bridge and wireless data acquisition system on the inspection vehicle (Kim and Kawatani 2009, Kim et al. 2008, Kim et al. 2011). Despite the non-stationary property of the traffic-induced vibration, the idea of using the traffic-induced vibration data of short span bridges in BHM is that the parameter identified repeatedly under moving vehicles could provide a pattern and give useful information to make a decision about the bridge health condition. Many studies focus on changes of system frequencies and structural damping constants for bridge diagnosis, which are estimated utilizing a linear time-series model (e.g. Nair et al. 2006, Magalhaes and Cunha 2011, Kim et al. 2012). Since the 1970s, the use of state-space models for modal parameter identification in time-domain has been increasing and also has yielded new approaches. Gersch et al. (1973), for example, used the time series of an autoregressive moving average (ARMA) process to describe the random response of a vibrating structure to a white noise excitation. Shinozuka et al. (1982) obtain a second-order ARMA model to represent a vibrating structure in order to identify the structural parameters directly. Hoshiya and Saito (1984) include the parameters to be identified as additional state variables in the state vector using extended Kalman filter. These approaches regard the ambient vibration responses as random process of ARMA. Estimating the coefficients of ARMA model is a kind of nonlinear approach because both of the coefficients relating to AR and MA processes are unknown variables. Fortunately, the AR model with an infinite order is equivalent to the ARMA model, which means that one can express the responses of a linear system subjected to white-noise input using the AR model with sufficient large order (Wang and Fang, 1986, Xia and De Roeck, 1997).

From the view of utilizing a vehicle-bridge interactive system in the damage identification of bridges, a limited number of studies have been conducted. Through an analytical study, Kim and Kawatani (2008) show that if the moving wheel loads of 
the inspection vehicle are measured and the time histories of bridge responses and vehicle wheel loads can be synchronized, it will be possible to obtain more accurate damage identification by solving an inverse problem. Zhan et al. (2011) focus on damage identification using train-induced vibrations and sensitivity analysis for the nondestructive evaluation of railway bridges. However these studies are confined to analytical investigations.

Although existing research focusing on the use of a passing vehicle for BHM and damage detection of bridges has its own problems to be solved in terms of practical applications, one way to realize the drive-by inspection may be to make use of a mutual complementation of existing methods. In other words, it would be a smart way to integrate the three possible approaches - utilizing the inspection vehicle as an actuator, data acquisition and message carrier - into a single framework of BHM. Another remaining problem is to verify validity of the method experimentally, since the success of vibration-based BHM strongly depends on how to treat unknown factors in real vibration data, which are generally different from the white noise used in analysis, and the results derived from the data.

In this study, therefore, three levels of bridge condition screening based on drive-by monitoring are presented, and the feasibility of each approach is examined through independent scaled laboratory experiments. This study firstly summarizes the methodology used in the three levels of condition screening based on the drive-by inspection, and describes the independent moving vehicle laboratory experiments. Finally, the feasibility of each level of screening is discussed.

\section{Methodology}

Three major approaches for the drive-by bridge inspection are the level 1 screening method which monitors the bridge frequency estimated from the vehicle's vibration data, the level 2 screening method based on modal parameter identification using vibration data transmitted from bridges to the inspection vehicle, and the level 3 screening method for damage identification which uses data both from bridges and the inspection vehicle as shown in Fig. 1.

It is noteworthy that the level 1 screening is adopted for a rapid health screening tool with sacrifice of the accuracy. The level 2 screening, expecting to offer better information than the level 1 screening, focuses on changes of identified frequencies, damping constants and mode shapes of bridges. The level 3 screening is adopted to identify damage location and severity when some diagnostic symptoms are detected through level 1 or level 2 screening. It is expected that both the Level 1 and Level 2 
screenings do not need to block traffic. However, the Level 3 screening needs to block traffic. Otherwise, the accuracy of the damage identification will be poor, or a false identification will occur due to influences from random traffic.

\section{Level 1 Screening}

- Indirect method utilizing Inspection vehicle as an Actuator

\& Message carrier (sensor).

- It only utilizes vehicle responses for structural diagnosis.

(a)

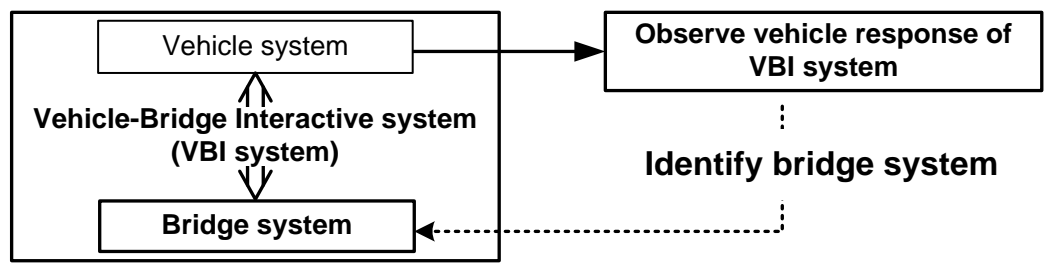

Level 2 Screening

- Direct method utilizing Inspection vehicle as an Actuator \& Data Acquisition System.

- It only utilizes bridge responses for structural diagnosis.

(b)

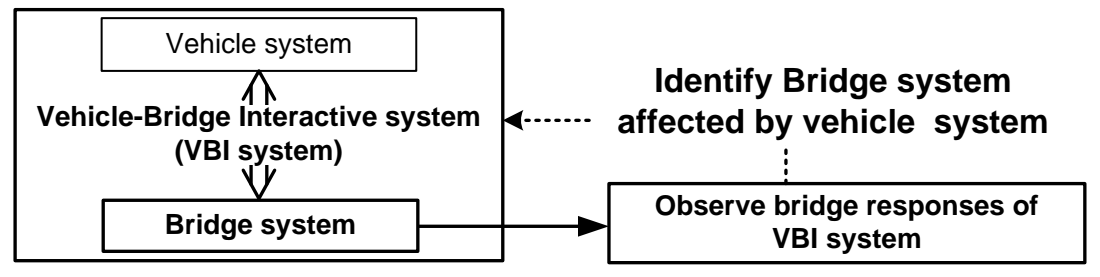

Level 3 Screening

- Direct method utilizing Inspection vehicle as an Actuator

\& Data Acquisition System.

- It utilizes both bridge and vehicle responses to detect severity

\& location of damages.

(c)

\begin{tabular}{|c|c|}
\hline Vehicle system & $\begin{array}{l}\text { Observe vehicle response of } \\
\text { VBI system }\end{array}$ \\
\hline $\begin{array}{c}\text { Vehicle-Bridge Înteractive system } \\
\text { (VBI system) }\end{array}$ & $\begin{array}{c}\text { bBI system } \\
\vdots \\
\vdots \\
\end{array}$ \\
\hline Bridge system & $\begin{array}{l}\text { Observe bridge responses of } \\
\text { VBI system }\end{array}$ \\
\hline
\end{tabular}

Fig. 1 Scheme of the drive-by bridge inspection: (a) Level 1 screening utilizing only vehicle responses; (b) Level 2 screening utilizing only bridge responses; and (c) Level 3 screening utilizing both vehicle and bridge responses.

The theoretical feasibility of all methods can be explained using the dynamic equations of the bridge-vehicle interactive system shown in Fig. 2. To make the problem simple a 2DOF vehicle model is considered as shown in Fig. 2, where $z_{v}(t)$ and $\theta_{v y}(t)$ represent vehicle's bounce and pitching motions respectively. In that figure, $m_{v}$ denotes the vehicle mass. Additionally, $k_{v s}$ and $c_{v s}$ denote the spring constant and damping coefficient at the $s$-th axle of the vehicle respectively. The subscript $s$ 
indicates the position of an axle: that is, $s=1$ and $s=2$ respectively signify the first (or front) and second (or rear) axles. Distances from the vehicle's center of gravity to respective axles are denoted by $\lambda_{x 1}$ and $\lambda_{x 2} . z_{0}\left(x_{s}(t)\right)$ indicates the roadway surface roughness at a position of $x_{s}(t)$ from the bridge entrance which is assumed as the reference position.

Equations of motion for the 2DOF vehicle can be formulated as shown in Eqs. (1) and (2). Therein $w\left(x_{s}(t), t\right)$ represents the time-variant displacement of the bridge at the contact point of the tire located $x_{s}(t)$ from the reference position.

$$
\begin{aligned}
& m_{v} \ddot{z}_{v}(t)+\sum_{s=1}^{2} c_{v s}\left(\dot{z}_{v}(t)-(-1)^{s} \lambda_{x s} \dot{\theta}_{v y}(t)-\left(\dot{w}\left(x_{s}(t), t\right)-\dot{z}_{0}\left(x_{s}(t)\right)\right)\right) \\
& +\sum_{s=1}^{2} k_{v s}\left(z_{v}(t)-(-1)^{s} \lambda_{x s} \theta_{v y}(t)-\left(w\left(x_{s}(t), t\right)-z_{0}\left(x_{s}(t)\right)\right)\right)=0 \\
& m_{v} \lambda_{x 1} \lambda_{x 2} \ddot{\theta}_{v y}(t) \\
& -\sum_{s=1}^{2}(-1)^{s} \lambda_{x s} c_{v s}\left(\dot{z}_{v}(t)-(-1)^{s} \lambda_{x s} \dot{\theta}_{v y}(t)-\left(\dot{w}\left(x_{s}(t), t\right)-\dot{z}_{0}\left(x_{s}(t)\right)\right)\right) \\
& -\sum_{s=1}^{2}(-1)^{s} \lambda_{x s} k_{v s}\left(z_{v}(t)-(-1)^{s} \lambda_{x s} \theta_{v y}(t)-\left(w\left(x_{s}(t), t\right)-z_{0}\left(x_{s}(t)\right)\right)\right)=0
\end{aligned}
$$

Combining the interaction force at the contact point of a vehicle wheel with the dynamic equation of motion of a bridge provides equations of motion for the vehicle-bridge interactive system. The dynamic equation of a bridge under a moving vehicle is definable as

$$
\mathbf{M}_{b r} \ddot{\mathbf{q}}_{r}(t)+\mathbf{C}_{b r} \dot{\mathbf{q}}_{r}(t)+\mathbf{K}_{b r} \mathbf{q}_{r}(t)=\sum_{s=1}^{2} \boldsymbol{\psi}_{s}(t) P(t)
$$

where $\mathbf{M}_{b r}, \mathbf{C}_{b r}$ and $\mathbf{K}_{b r}$ respectively represent the mass, damping, and stiffness matrices of the bridge. $\mathbf{q}_{r}(t)$ is the displacement vector; over dots denote derivatives with respect to time. $\psi_{s}(t)$ is a load distribution vector to each node of the element on which a tire contacts. $P_{s}(t)$ in Eq. (3) denotes the wheel load at a tire and is definable as

$$
P_{s}(t)=\left(1-\frac{\lambda_{x s}}{\lambda_{x}}\right) m_{v} g+c_{v s} \dot{\delta}_{s}(t)+k_{v s} \delta_{s}(t)
$$

where, $\delta_{s}(t)$ denotes the relative vertical displacement at the $s$-th axle of the vehicle and is definable as

$$
\delta_{s}(t)=z_{v}(t)-(-1)^{s} \lambda_{x s} \theta_{v y}(t)-\left\{w\left(x_{s}(t), t\right)-z_{0}\left(x_{s}(t)\right)\right\}
$$




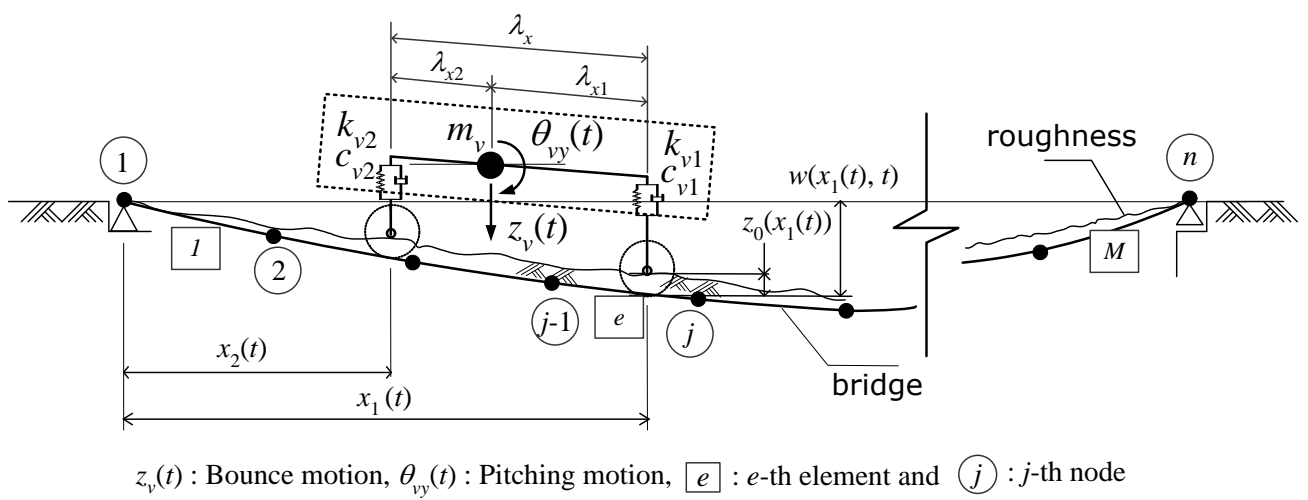

Fig. 2. Scheme of a bridge-vehicle interactive system in moving vehicle laboratory. experiment.

A goal for the level 1 screening is extracting changes of bridge's dynamic features from the vehicle vibrations since dynamic equations of motion of the vehicle traveling on a bridge clearly contain bridge's responses $w\left(x_{s}(t), t\right)$ as shown in Eqs. (1) and (2). This means that if the amplitude of the bridge response is big enough then detecting bridge's frequencies becomes feasible theoretically.

Both level 2 and level 3 screenings basically rely on bridges' vibration data actuated by the inspection vehicle travelling on the bridge. The discrepancy between the two methods is in the use of external forces generated by the moving vehicle. In other words, the level 2 screening is an output only method. On the other hand, the level 3 screening needs both the vibration data of the bridge and the vehicle's dynamic wheel loads.

The eigen system realization algorithm (ERA) (Pappa and Ibrahim (1981)) based on the state space equation of the dynamic system is adopted for the level 2 screening. Many studies focus on changes of system frequencies and structural damping constants for bridge diagnosis, which are estimated utilizing a linear time-series model (e.g. Gersch et al. 1973, Shinozuka et al. 1982, Hoshiya and Saito 1984, Wang and Fang 1986, Xia and De Roeck 1997, Kim et al. 2010).

From Eq. (3) the state vector $\mathbf{x}(t)$ for the equation of motion of a bridge under a moving vehicle is definable as

$$
\mathbf{x}(t)=\left[\begin{array}{c}
\mathbf{q}_{r}(t) \\
\dot{\mathbf{q}}_{r}(t)
\end{array}\right]
$$

If $\mathbf{y}(t) \in \mathbf{R}^{m}$ denotes output of the bridge taken from $m$ observation points, then the corresponding state equation of a continuous-time system is described as 


$$
\begin{aligned}
\dot{\mathbf{x}}(t) & =\mathbf{A x}(t)+\mathbf{B w}(t) \\
\mathbf{y}(t) & =\mathbf{C x}(t)
\end{aligned}
$$

where, $\mathbf{A}, \mathbf{B}$ and $\mathbf{C}$ respectively denote system, input influence and output influence matrices. Especially, $\mathbf{C}$ is a transformation matrix mapping the position of system degrees of freedom with measured outputs which consists of zero or one. $\mathbf{w}(t)$ denotes external effects (or noise term) for the system.

Linear dynamic system can be identified using the AR model as (e.g. Kim et al., 2012)

$$
y(k)+\sum_{i=1}^{p} a_{i} y(k-i)=e(k)
$$

where, $y(k)$ denotes output of the system, $a_{i}$ is the $i$-th order AR coefficient and $e(k)$ indicates the noise term.

To estimate AR parameters, the autocorrelation function of $y(k)$ which is obtainable by multiplying each term of Eq. (9) with $y(k-s)$ and taking mathematical expectation is used. This process yields the following Yule-Walker equation.

$$
\mathbf{R a}=-\mathbf{r}
$$

where $\mathbf{R}$ is a Toeplitz matrix about $R(p, s)=\mathrm{E}[y(k-p) y(k-s)]$ which is the autocorrelation function of the signal. $\mathbf{a}=\left[a_{1} ; \ldots ; a_{\mathrm{n}}\right]$ and $\mathbf{r}=\left[R_{1} ; \ldots ; R_{\mathrm{n}}\right]$.

The Levinson-Durbin algorithm is adopted to solve Eq. (10). It is noteworthy that the coefficient $a_{p}$ is a pole of the system because the $z$-transformation of Eq. (9) is writable as

$$
Y(z)=H(z) E(z)=\frac{1}{1+\sum_{i=1}^{p} a_{i} z^{-i}} E(z)
$$

where $Y(z)$ and $E(z)$ are $z$-transformation of $y(k)$ and $e(k), H(z)$ is the transfer function of the system in the discrete-time complex domain, and $z^{-i}$ denotes the forward shift operator.

Values of $z$ in which the elements of the transfer function matrix show infinite values are the pole. In other words, the denominator of the transfer function is the characteristic equation of the dynamic system as

$$
z^{n}+a_{1} z^{n-1}+a_{2} z^{n-2}+\cdots+a_{n-1} z+a_{n}=0
$$

The poles on the complex plane are related with the frequency and damping constant of the dynamic system as (Papas and Ibrahim 1981) 


$$
z_{k}=x_{\mathrm{Re}}^{k} \pm j x_{\mathrm{Im}}^{k}=\exp \left(-h_{k} \omega_{k} \pm j \omega_{k} \sqrt{1-h_{k}^{2}}\right)
$$

where, $h_{k}$ and $\omega_{k}$ are the damping constant and circular frequency of $k$-th mode of the system. $j$ represents the imaginary unit.

Therefore, the frequency and damping constant can be obtained from the following equations.

$$
\begin{aligned}
& \omega_{k} \sqrt{1-h_{k}^{2}}=\frac{1}{T} \tan ^{-1}\left(\frac{x_{\mathrm{Im}}^{k}}{x_{\mathrm{Re}}^{k}}\right) \\
& h_{k} \omega_{k}=-\frac{1}{T} \ln \left(\sqrt{\left(x_{\mathrm{Re}}^{k}\right)^{2}+\left(x_{\mathrm{Im}}^{k}\right)^{2}}\right)
\end{aligned}
$$

It should be noted that operational traffic-induced vibrations of bridges are not due to white noise excitation actually, but nevertheless the idea of the level 2 screening using traffic-induced vibrations of short span bridges for system identification is that repeatedly identified system parameters under a given moving vehicle can provide a pattern or even a statistical one which may give useful information to make a decision about the bridge's health condition.

The concept of the level 3 screening is based on the fact that the stiffness distribution in the structure changes as a result of damage. This change is detectable by measuring dynamic responses in the inspection vehicle whose dynamic wheel loads or dynamic properties are known. The linear equation for the bridge's structural stiffness can also be derived from Eq. (3) as shown in Eq. (16) which is a pseudo-static formulation showing change of structural stiffness (Kim and Kawatani 2008).

$$
\mathbf{K}_{b r} \mathbf{q}_{r}(t)=\mathbf{f}(t) ; \mathbf{f}(t)=\sum_{s=1}^{2} \boldsymbol{\psi}_{s}(t) P_{s}(t)-\mathbf{M}_{b r} \ddot{\mathbf{q}}_{r}(t)-\mathbf{C}_{b r} \dot{\mathbf{q}}_{r}(t)
$$

The change of stiffness $\mathbf{K}_{b r}$ in Eq. (16) indicates a change in the bridge's stiffness due to damage. Detecting the change in $\mathbf{K}_{b r}$ is the basic concept of the damage identification proposed for the level 3 screening. The change of the element stiffness is also obtainable using the element stiffness index (ESI) as

$$
\mu_{e}=\frac{\mathbf{K}_{d}}{\mathbf{K}_{i}}
$$

where $\mu_{e}$ is the element stiffness index, and $\mathbf{K}_{i}$ and $\mathbf{K}_{d}$ signify the stiffness of the $e$-th element of an intact and damage states, respectively. 
Estimating the ESI is the final goal for the level 3 screening. A noteworthy point is that the ESI value is unity for the intact, i.e. healthy, state of an element. This means that the value is less than unity for damaged elements. Kim and Kawatani (2008) show details of the methodology.

Table 1 Bridge model properties

\begin{tabular}{c|c|c|ccc}
\hline Span & Material & Cross & \multicolumn{4}{|c}{ Natural frequency $(\mathrm{Hz})$} & \\
Length & density & sectional & first & third & Damping Ratio \\
$L(\mathrm{~m})$ & $w\left(\mathrm{~kg} / \mathrm{m}^{3}\right)$ & area, $A\left(\mathrm{~m}^{2}\right)$ & $f_{b, 1}$ & $f_{b, 3}$ & for $1^{\text {st }}$ mode, $\xi$ \\
& & & & & \\
\hline 5.4 & 7800 & $6.7 \times 10^{-3}$ & 2.69 & 23.4 & 0.016 \\
\hline
\end{tabular}

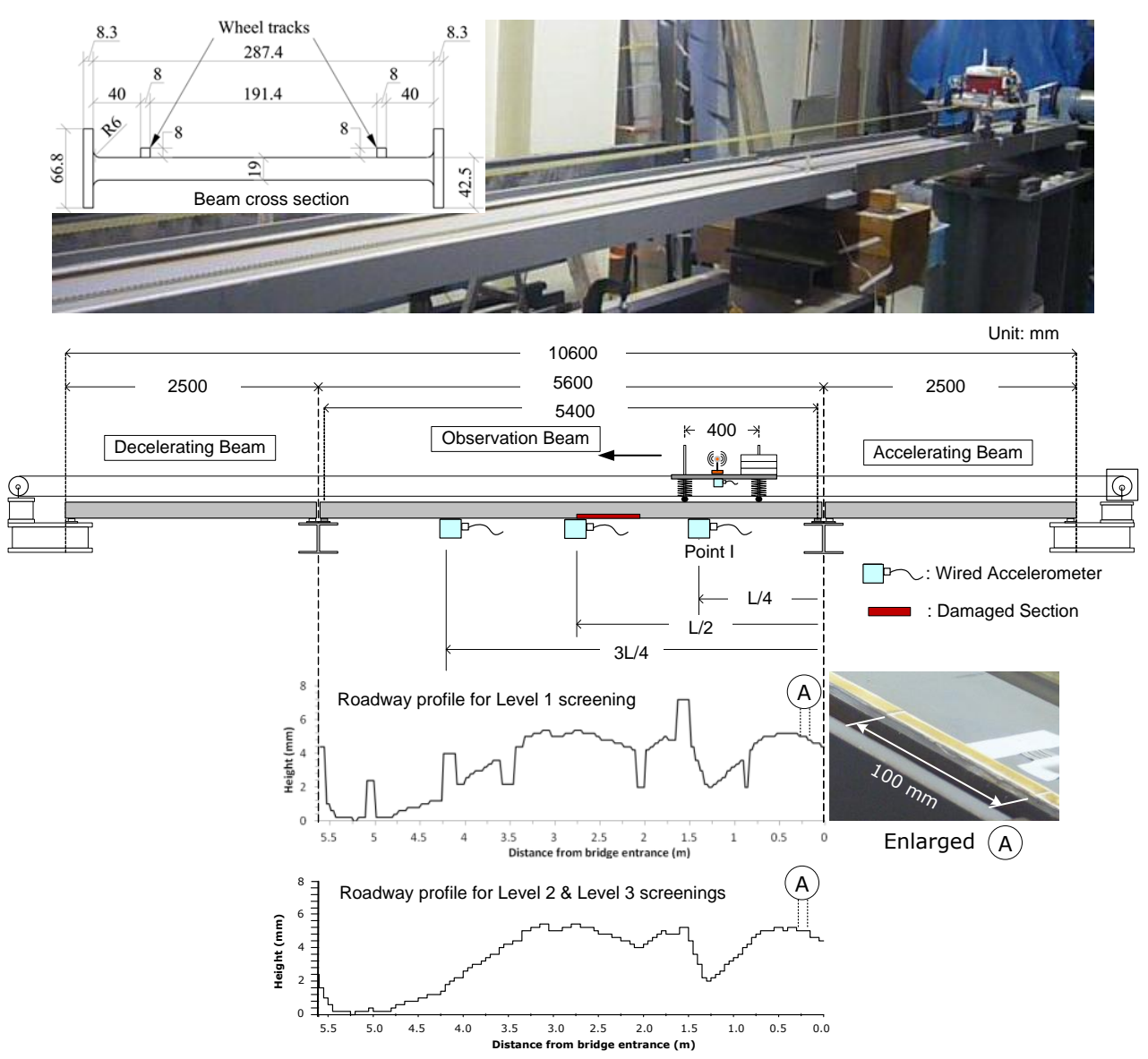

Fig. 3. Experiment setup, observation points and roadway profiles for level 1 screening and for level 2 and level 3 screenings. 


\section{Laboratory Experiment}

A scaled moving vehicle laboratory experiment is performed to investigate feasibility of the drive-by health monitoring approach. The scaled bridge model used in the experiment is a $5.4 \mathrm{~m}$ simply supported steel beam. It is fitted with accelerometers and displacement transducers at quarter span, mid-span and three-quarter span to monitor its response in free vibration tests and during crossings of the vehicle over the bridge. The beam properties obtained from the manufacturer and free vibration tests are given in Table 1.

The experiment setup and roadway profiles considered in the experiment are shown in Fig. 3 in which three simple beams for accelerating, decelerating and observation are used. Roadway profiles were considered in the experiment as exist on actual bridges. Both left and right wheel paths of the vehicle were paved with an electrical tape at the interval of $100 \mathrm{~mm}$ as shown in Fig. 3. The thickness of the tape was $0.2 \mathrm{~mm}$. In the experiment for level 1 screening, a rougher roadway than that adopted in level 2 and level 3 screenings is considered in order to provide a higher level of excitation for the experimental vehicle. Damage scenarios with artificial damage in the bridge are summarized in Fig. 4.

a)

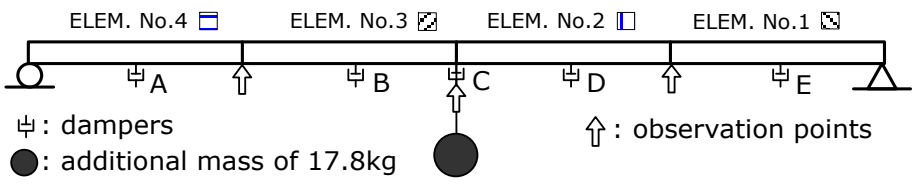

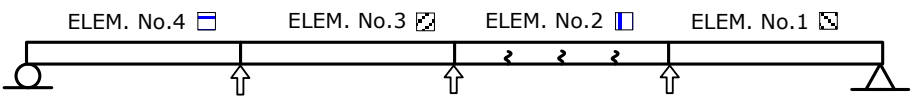

(b)

\&\& : saw cuts iv: Observation points

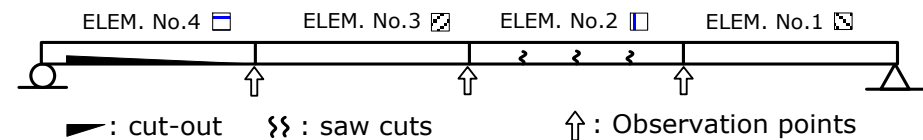

(c)
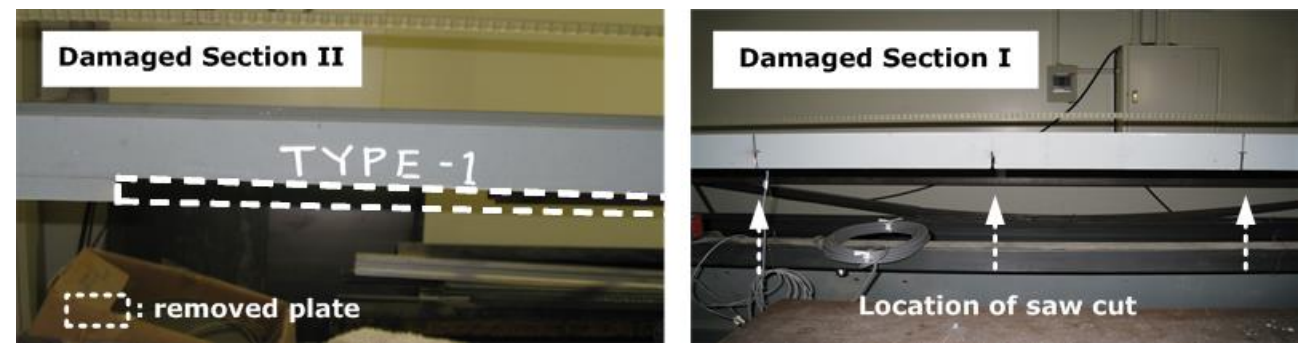

Fig. 4. Damage scenarios: (a) for level 1 screening; (b) for level 2 and level 3 screenings with damage scenario of D1; (c) for level 2 and level 3 screenings with damage scenario of D2; and (d) photos of two artificial damage types. 
In the experiment which examines the feasibility of the level 1 screening, the damping of the bridge is varied by applying old displacement transducers at particular points on the bridge in addition to a mass of $17.8 \mathrm{~kg}$ added at midspan. The layout of these transducers is illustrated in Fig. 4(a), in which the dampers are denoted by the letters, A to E. The old transducers are used as they provide frictional resistance to bridge displacements at the chosen locations. The damping constant changes from $1.4 \%$ for the initial case to $2.1 \%$ and $4.3 \%$ due to an additional damper at the span center and five additional dampers respectively. The additional mass is used to adjust the frequency of the bridge as frequently damage which causes changes in damping may cause some change in frequency. The additional mass causes change of the natural frequency for the first bending mode from $2.7 \mathrm{~Hz}$ to $2.5 \mathrm{~Hz}$.

Two damage scenarios are considered in the experiment to investigate feasibility of the level 2 and level 3 screenings: for the first damage scenario (hereafter D1), three saw cuts are applied to both sides of web plates at ELEM No.2 of the bridge; the second damage scenario (hereafter D2) considers both saw cuts at ELEM No.2 and cut-out at ELEM. No.4. For damage scenario D1, about $11 \%$ loss of the bending rigidity of the bridge is observed. About $23 \%$ loss of bending rigidity of the damaged bridge is observed due to damage scenario D2. Damage changes the natural frequencies of the bridge: $2.6 \mathrm{~Hz}$ under damage scenario D1; and $2.5 \mathrm{~Hz}$ under damage scenario under damage scenario D2. Apparently the damage causes a decrease in natural frequencies. It should be noted that the bridge structure and damage types performed herein are representatives for illustration only, but not confined to specific types. In this feasibility study, the focus is put on verifying the feasibility of the present approach. Therefore, the artificial damage is not intended to perfectly simulate real damage, but to make the bridges serve as damaged samples in comparison to intact ones, in terms of bending rigidity reduction.

A scaled two-axle vehicle model is instrumented for the experiments as shown in Fig. 5. It is fitted with 2 accelerometers to monitor the vehicle bounce motion; these are located at the center of the front and rear axles respectively. It also includes a wireless router and data logger which allow the acceleration data to be recorded remotely. The vehicle model can be adjusted to obtain different axle configurations and dynamic properties; the spring stiffness of the axles can be varied by changing the springs while the body mass can be varied using steel plates. The properties of the three vehicle model configurations chosen for these experiments are given in Table 2, which were determined prior to testing. 
Table 2 Vehicle model properties

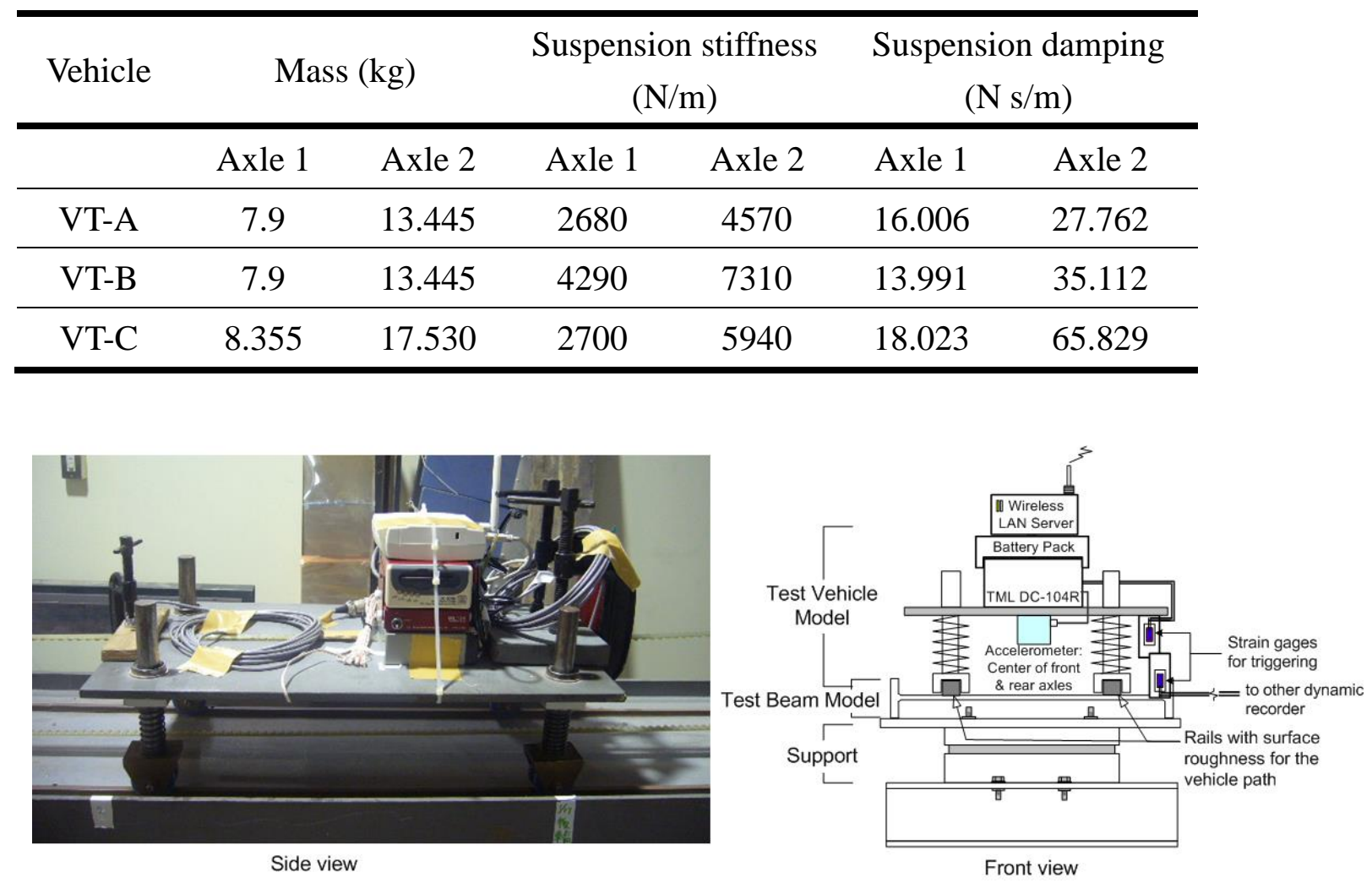

Fig. 5. Experimental vehicle.

Three different vehicle models, of which the natural frequency of the bounce motion can be varied using different sets of masses and springs, are considered in the experiment. The three vehicles, called VT-A, VT-B and VT-C, are used in the experiment. Natural frequencies for the bounce motion of these vehicle models are $2.93 \mathrm{~Hz}, 3.76 \mathrm{~Hz}$ and $3.03 \mathrm{~Hz}$ respectively.

The speed of the vehicle was maintained constant by an electronic controller as it crossed the bridge. The entry and exit of the vehicle to the beam was monitored using strain sensors. Two different scaled vehicle speeds of $S 1=0.93 \mathrm{~m} / \mathrm{s}$ and $\mathrm{S} 2=1.63 \mathrm{~m} / \mathrm{s}$ are considered in order to investigate the effect of the vehicle speed on the screening results. Therefore, six traffic scenarios are considered: SCN1 of VT-A vehicle traveling with speed of S1; SCN2 of VT-A vehicle traveling with speed of S2; SCN3 of VT-B vehicle traveling with speed of S1; SCN4 of VT-B vehicle traveling with speed of S2; SCN5 of VT-C vehicle traveling with speed of S1; and SCN6 of VT-C vehicle traveling with speed of S2.

$\mathrm{S} 1$ and S2 give speed parameters $(\alpha)$ of 0.032 and 0.056 respectively using Eq. (18). They are similar to speed parameters of 0.029 and 0.059 estimated using speeds 
of $20 \mathrm{~km} / \mathrm{h}$ and $40 \mathrm{~km} / \mathrm{h}$ respectively for an existing $40.4 \mathrm{~m}$ bridge span with first bending mode of $2.35 \mathrm{~Hz}$.

$$
\alpha=\frac{v}{2 f_{b, 1} L}
$$

In Eq. (18), $\alpha$ is the speed parameter, $v$ is the vehicle speed $(\mathrm{m} / \mathrm{s}), f_{b, 1}$ is the first natural frequency of the bridge $(\mathrm{Hz})$ and $L$ is the bridge span length $(\mathrm{m})$. This dimensionless parameter is important for the scaling of the experimental model as it is used to maintain a relationship between vehicle speed, frequency and span length for the $5.4 \mathrm{~m}$ beam which is similar to that for a $40.4 \mathrm{~m}$ bridge subject to real traffic.

Two different speeds of $\mathrm{S} 1=0.93 \mathrm{~m} / \mathrm{s}$ and $\mathrm{S} 2=1.63 \mathrm{~m} / \mathrm{s}$ are considered in order to investigate the effect of the vehicle speed on the screening results.

\section{Condition Screening}

\subsection{Level 1 screening}

Fig. 6 shows an example of the spectra of bridge accelerations obtained from crossings of the vehicle over the bridge in the experiment for varying vehicle properties and vehicle speed. It can be seen in Fig. 6(a) that the bridge peak occurs at $2.54 \mathrm{~Hz}$ for the data corresponding to VT-B and S1. The bridge peaks occurring for VT-A and VT-C occur at $2.54 \mathrm{~Hz}$ and $2.44 \mathrm{~Hz}$ respectively but are difficult to distinguish in this figure. It can be seen in Fig. 6(b) that for vehicle VT-A, the bridge peak frequency and magnitude increase with increasing speed.

Fig. 7 shows the spectra of vehicle accelerations corresponding to the experimental bridge measurements shown in Fig. 6(a) for speed S1 and varied vehicle properties. The spectral resolution is $\pm 0.098 \mathrm{~Hz}$ in this figure. Axle 2 detects the bridge frequency much better than axle 1; although both axles experience peaks at $2.44 \mathrm{~Hz}$ with similar PSD magnitude. This is due to the relative dominance of the bridge frequency peak in the spectra, which is caused by the greater axle load of axle 2. The greater load reduces the magnitude of the bouncing and pitching response at this axle due to the road profile excitation thus increasing the influence of the bridge model on its vibration. As axle 1 is lighter, it is much more sensitive to bouncing and pitching due to the road profile, resulting in the large peaks at $3.91 \mathrm{~Hz}$ and $4.7 \mathrm{~Hz}$, which correspond to vehicle frequencies. This can be seen by comparing the magnitudes of the acceleration spectra for axle 1 and axle 2 in Fig. 7. 
It can be seen that the 2nd axle of vehicle VT-C (Fig. 7(b)), is excellent for frequency detection as the bridge vibration dominates the vehicle spectra with a clear peak at $2.44 \mathrm{~Hz}$. Studying this figure, it can be seen that vehicles VT-A and VT-C provide the best opportunity for frequency detection as clear bridge peaks are the most dominant in the PSD for axle 2. This suggests that using vehicles with axle bounce frequencies close to the bridge natural frequency, such as VT-A and VT-C here, is beneficial for frequency detection. For VT-B, the higher pitch $(5.1 \mathrm{~Hz})$ and bounce frequency $(3.51 \mathrm{~Hz})$ also dominate the spectra, reducing the influence of the bridge vibration on the vehicle response.
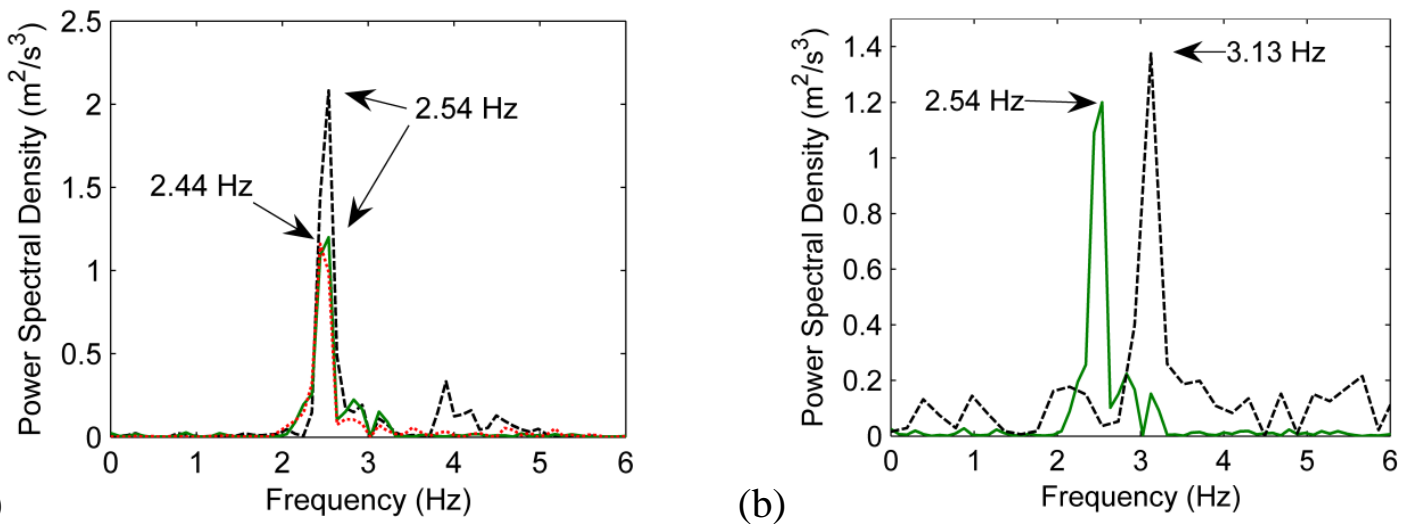

Fig. 6. Spectra of bridge midspan accelerations varying (a) Vehicle properties; (-) VT-A and S1, (--) VT-B and S1, (...) VT-C and S1 and (b) Speed; (-) VT-A and S1, (--) VT-A and S2.
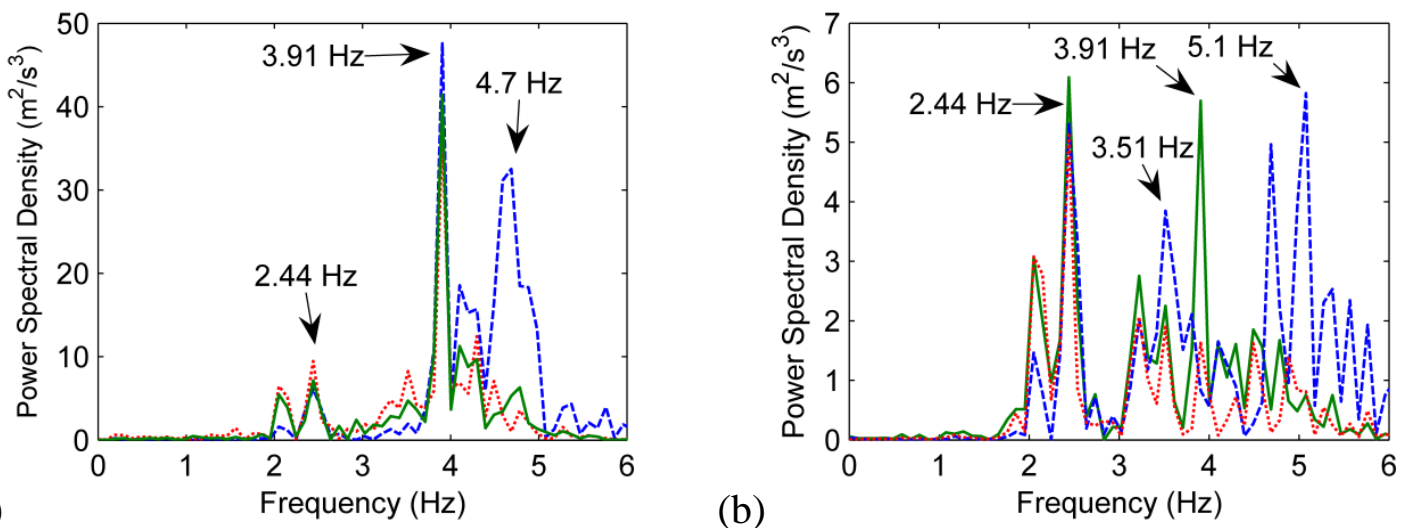

Fig. 7. Spectra of vehicle accelerations for speed S1: (a) Axle 1 (b) Axle 2; (-) VT-A, (--) VT-B, (•.) VT-C.

Fig. 8 shows the spectra of vehicle accelerations obtained from the experiment for vehicle VT-A crossing the bridge at speeds S1 and S2 respectively; S1 has already 
been presented in Fig. 7 but is included here for comparison. The corresponding spectral resolutions are $\pm 0.049 \mathrm{~Hz}$ and $\pm 0.195 \mathrm{~Hz}$ respectively. For each speed the bridge frequency peak (Fig. 6(b)) is detected in the vehicle spectra, with axle 2 having a greater sensitivity once again. Vehicle acceleration spectra magnitude increases with vehicle speed. For the higher speed $\mathrm{S} 2(=1.63 \mathrm{~m} / \mathrm{s})$, the bridge frequency peak occurs at $3.13 \mathrm{~Hz}$ (Fig. 6(b)). This appears to be caused by a combination of reduced spectral resolution and the interaction of the vehicle and bridge models at this speed. However, it is still detected clearly by axle 2 . All vehicles show similar trends with speed and in particular, speed $\mathrm{S} 1(=0.93 \mathrm{~m} / \mathrm{s})$ provides the best results due to its compromise between spectral resolution and bridge excitation.
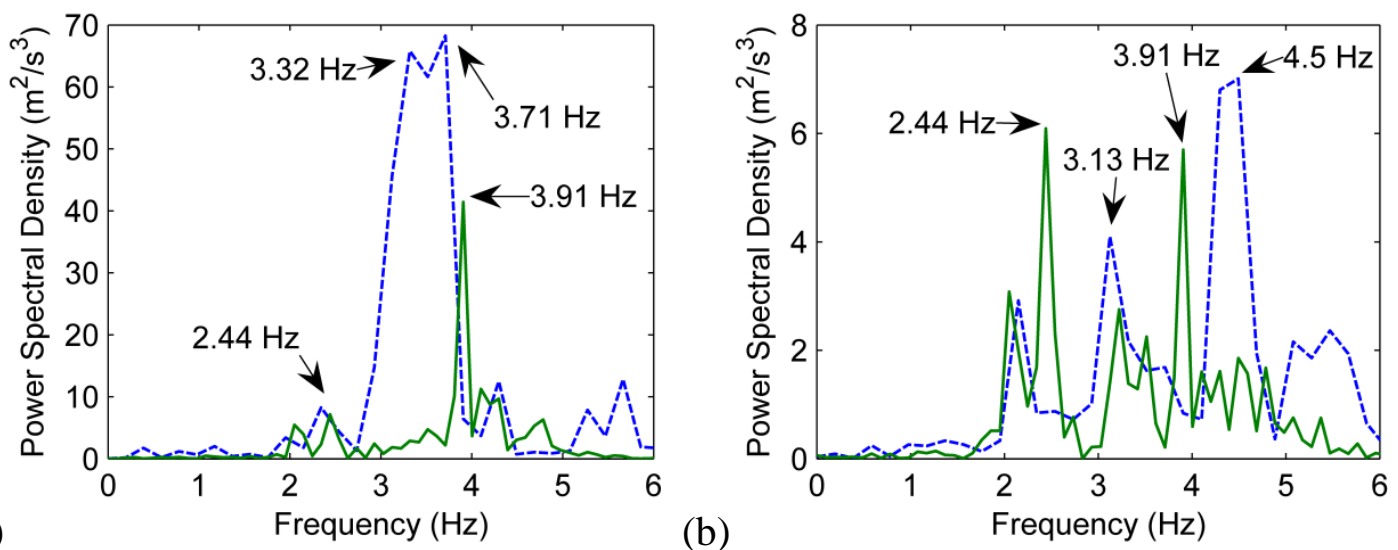

Fig. 8. Spectra of accelerations for vehicle VT-A: (a) Axle 1 (b) Axle 2; (-) speed $\mathrm{S} 1,(--)$ speed $\mathrm{S} 2$.

(a)

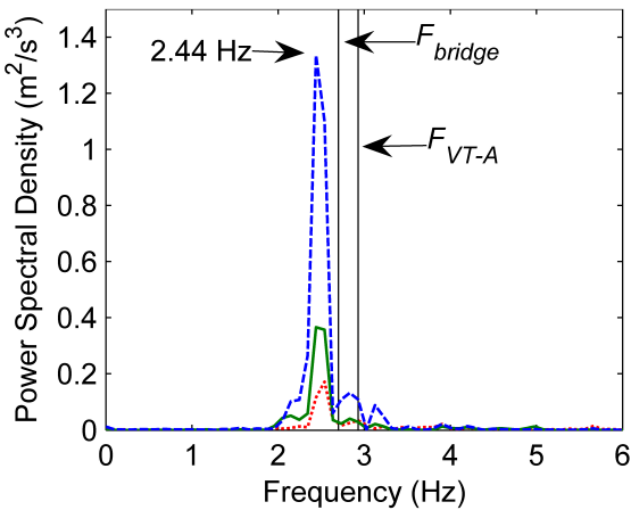

(b)

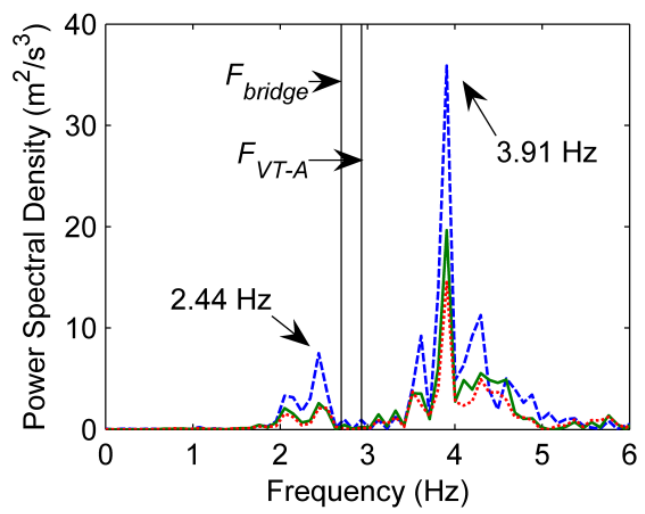

Fig. 9. Spectra of mean acceleration responses: VT-A for speed S1 of $0.93 \mathrm{~m} / \mathrm{s}$ : (a) bridge midspan; and (b) front axle of vehicle. Scenarios: (---) Intact, $(-)$ C, $(\cdots)$ ABCDE. 
Fig. 9 compares the mean acceleration spectra of the model bridge and vehicle obtained from all scenarios for 5 crossings of vehicle VT-A at speed S1 over the bridge. The bridge frequency peak at $2.44 \mathrm{~Hz}$ occurs in both Figs. 9(a) and (b). It can be seen that as the damping increases, i.e., from the 'Intact' scenario to scenarios with dampers at locations ' $C$ ' and 'ABCDE' respectively (Fig. 4(a)), the peak magnitude at the bridge frequency in both the bridge and vehicle spectra decreases. In Fig. 9(a), the percentage decreases in peak magnitude from the intact scenario to scenarios $\mathrm{C}$ and $\mathrm{ABCDE}$ are $70.3 \%$ and $88.2 \%$ respectively. In Fig. 9(b), although the peak at $2.44 \mathrm{~Hz}$ in the vehicle spectra is not the dominant peak, it follows the trend with decreases of $65.8 \%$ and $72.5 \%$ respectively. Furthermore, this trend also occurs at the peak in the vehicle spectra at $3.91 \mathrm{~Hz}$, which corresponds to the body pitch frequency of the vehicle. The percentage changes at this peak in Fig. 9(b) are $45.2 \%$ and $59.6 \%$ respectively. These results highlight the feasibility of detecting changes in bridge damping from vehicle vibrations. It is noteworthy that the dominant frequency of the bridge in Fig. 9(a) is biased from the natural frequencies of the bridge and vehicle, which shows difficulty in distinguishing the bridge's natural frequency from traffic-induced vibrations.

For all scenarios investigated but omitted in this paper, the bridge frequency was identified in the vehicle spectra. The results observed in this experiment show that the frequency peak and its magnitude, detected from the response of the vehicle as it crosses over the bridge, will vary depending on the vehicle and its speed, therefore speed selection is deemed to be a critical factor in frequency detection. The higher speed, $\mathrm{S} 2=1.63 \mathrm{~m} / \mathrm{s}$, provides larger magnitude peaks in the spectra but the spectral resolution is not as high as for speed S1. For VT-A vehicle and speed of S1, changes in damping are detected in the vehicle spectra. These results indicate that to confirm the feasibility of the system, further investigation of vehicle configuration, speed and bridge model damping scenarios is necessary. However, those approaches are feasible for extracting the natural frequency of bridges within restricted conditions.

\subsection{Level 2 screening}

System parameters of the intact and damaged model bridges are identified by the AR model (Kim et al. 2012). The change of identified system parameters such as dominant frequencies and corresponding damping constants is investigated. The reason to take notice of dominant dynamic parameters is that traffic-induced bridge vibrations are usually affected by vehicle's dynamic properties and the identified system parameters are not the bridge's but those of the vehicle-bridge interactive 
system. Therefore, this study investigates change of the dominant dynamic parameters due to damage rather than utilizing change of the natural modal parameters.

(a)
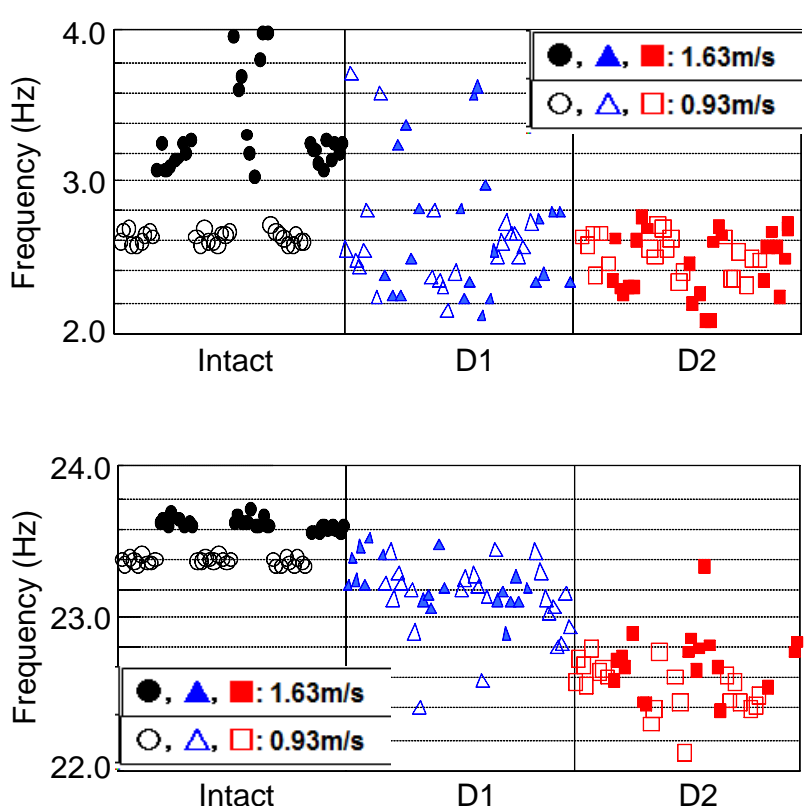

(b)

Fig. 10. Variation of identified frequencies for level 2 screening: (a) observed frequency near $4 \mathrm{~Hz}$; and (b) observed frequency near $23 \mathrm{~Hz}$.

Table 3 Statistical features of identified system frequency

\begin{tabular}{ccccc|ccc}
\hline & & & S1 & & \multicolumn{3}{c}{ S2 } \\
\hline & & Intact & D1 & D2 & Intact & D1 & D2 \\
\hline \multirow{3}{*}{ OF-A } & Mean & 2.63 & 2.62 & 2.53 & 3.34 & 2.67 & 2.46 \\
& SD & 0.041 & 0.369 & 0.123 & 0.290 & 0.444 & 0.207 \\
& CV & 0.015 & 0.141 & 0.049 & 0.087 & 0.166 & 0.084 \\
\hline \multirow{4}{*}{ OF-B } & Mean & 23.39 & 23.24 & 22.55 & 23.63 & 23.12 & 22.73 \\
& SD & 0.021 & 0.161 & 0.161 & 0.042 & 0.251 & 0.213 \\
& CV & 0.001 & 0.007 & 0.007 & 0.002 & 0.011 & 0.009
\end{tabular}

* OF-A : Observed Frequency near $2.7 \mathrm{~Hz}, \mathrm{OF}-\mathrm{B}:$ Observed Frequency near $23.4 \mathrm{~Hz}$, SD : Standard Deviation

Dominant system frequencies and damping constants are identified using the data taken from six traffic scenarios mentioned in the previous section 3. Identified dominant frequencies of the bridge model are summarized as shown in Figs. 10(a) and 10 (b) for the lower mode near $2.7 \mathrm{~Hz}$ and higher mode around $23 \mathrm{~Hz}$ respectively. Therein the solid circles, solid triangles and solid squares are identified parameters 
observed for the intact bridge, the bridge model with D1 damage and bridge model with D2 damage respectively under vehicle speed $\mathrm{S} 2=1.63 \mathrm{~m} / \mathrm{s}$. The symbols with no fill correspond to the identified results for vehicle speed $\mathrm{S} 1=0.93 \mathrm{~m} / \mathrm{s}$.

Observations demonstrate that the lower speed gives similar frequencies with those from free vibration experiment. The mean values of identified frequencies of the intact girder under $\mathrm{S} 1$ are $2.63 \mathrm{~Hz}$ and $23.4 \mathrm{~Hz}$ as shown in Table 3, which link with $2.69 \mathrm{~Hz}$ and $23.4 \mathrm{~Hz}$ for the first and third modes obtained from the free vibration respectively. Those mean frequencies after introducing the damage D1 are $2.62 \mathrm{~Hz}$ and $23.24 \mathrm{~Hz}$ and introducing the damage $\mathrm{D} 2$, they become $2.53 \mathrm{~Hz}$ and $22.55 \mathrm{~Hz}$ respectively. On the other hand, those identified results for the vehicle travelling at higher speed (S2) are greatly biased from parameters obtained from free vibrations as shown in Fig. 10. The results demonstrate that the effect of the vehicle system on bridge vibration, so called traffic-induced vibration of bridges or non-stationary vibration, increases with increasing speed, and as a result the identified results under higher vehicle speed yield more biased identification results than those of lower speed. For the first frequencies in any scenario, mean values tend to decrease while on the other hand the coefficients of variation (CVs) tend to increase due the damage.

A similar tendency is also observed in the identified damping constants summarized in Figs. 11(a) and 11(b) for the damping constants of lower mode near $2.7 \mathrm{~Hz}$ and a higher mode around $23 \mathrm{~Hz}$ respectively. The statistical parameters of the observed damping constants are summarized in Table 4. Usually the damping constants derived from eigenvalue of system matrix A may be subject to appreciable error (Pappa and Ibrahim 1981), and as a result larger coefficient of variance than that of the identified frequency is observed. However, despite their appreciable error the pattern change of identified damping constants due to the damage is very apparent comparing to that of the dominant frequency as shown in Fig. 11.

Observations from the identified results show that the damage causes disturbance of the identified dominant frequency and system damping constant regardless of vehicle speed and type. This is the reason why this study is focusing on the pattern change of mean value and $\mathrm{CV}$ of modal parameters to acquire additional information about current health condition of the bridge.

It also demonstrates that the identified system modal parameters, using the traffic-induced vibration data under a given moving vehicle such as an inspection car, can provide information for bridge's health condition. However, how to quantify and qualify the pattern changes for the structural diagnosis is a task that remains to be studied. 
(a)

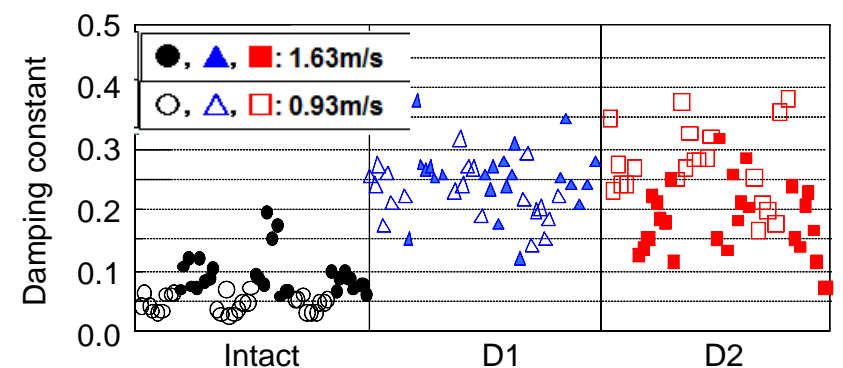

(b)

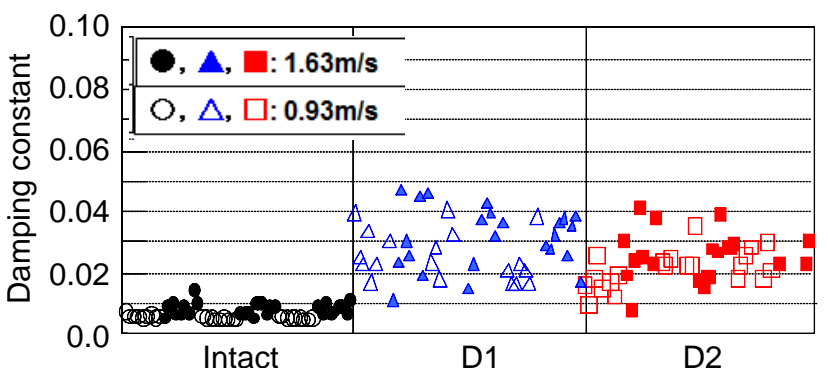

Fig. 11. Variation of identified damping constants for level 2 screening: (a) observed damping constant corresponding to the frequency near $4 \mathrm{~Hz}$; and (b) observed damping constant corresponding to the frequency near $23 \mathrm{~Hz}$.

Table 4 Statistical feature of identified system damping constant

\begin{tabular}{ccccc|ccc}
\hline & & \multicolumn{3}{c|}{ S1 } & \multicolumn{3}{c}{ S2 } \\
\hline & & Intact & D1 & D2 & Intact & D1 & D2 \\
\hline \multirow{4}{*}{ OD-A } & Mean & 0.0463 & 0.2371 & 0.2738 & 0.0944 & 0.2523 & 0.1863 \\
& SD & 0.0133 & 0.0662 & 0.0602 & 0.0331 & 0.0553 & 0.0571 \\
& CV & 0.288 & 0.279 & 0.220 & 0.351 & 0.219 & 0.306 \\
\hline \multirow{4}{*}{ OD-B } & Mean & 0.0054 & 0.0254 & 0.0214 & 0.0944 & 0.2523 & 0.1863 \\
& SD & 0.0006 & 0.0080 & 0.0058 & 0.0021 & 0.0097 & 0.0081 \\
& CV & 0.115 & 0.313 & 0.271 & 0.022 & 0.039 & 0.044 \\
\hline
\end{tabular}

* OD-A : Observed damping constant corresponding to frequency near $2.7 \mathrm{~Hz}$,

OD-B : Observed damping constant corresponding to frequency near $23.4 \mathrm{~Hz}$

\subsection{Level 3 screening}

This section discusses the feasibility of the level 3 screening which aims to identify damage. It should be noted that in each damage scenario, the element with the highest stiffness, i.e. the 'healthiest' element, relative to all other elements is chosen as the reference element and given an ESI value of unity. The ESI of all other elements are then normalized relative to this element. Identified ESI values for damage scenarios D1 and D2 are summarized in Fig. 12. ELEM. No. 4 and ELEM. 
No. 1 are identified as the healthiest elements in D1 and D2 respectively therefore their ESI values are set to unity.

For D1, the damage in element No.2 is well identified by the proposed method except in SCN3 (see Fig. 12(a)), the loading scenario 3, which identifies ELEM. No.1 as the element that is most damaged, rather than ELEM. No.2. Unfortunately, the reason for this unsuccessful identification in SCN3 is not yet clear. However, it is likely that the ESI's are reduced in ELEM. No.1 and 3 in all scenarios due to the bridge acting as a continuum system and the proposed method can still be regarded as an effective tool in identifying the most suspected damage element, say ELEM. No.2 in this damage case. The error related to identifying the damage severity is also shown in Fig. 12. The error varies up to $4.5 \%$, and it demonstrates the proposed method can also presume the damage severity.

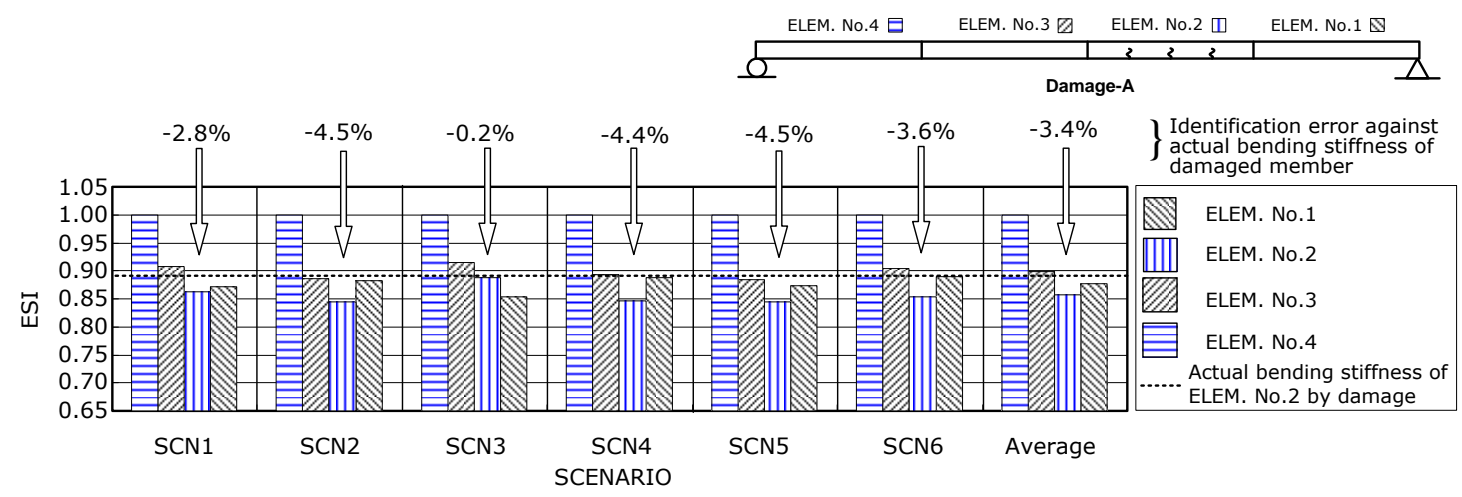

(a)

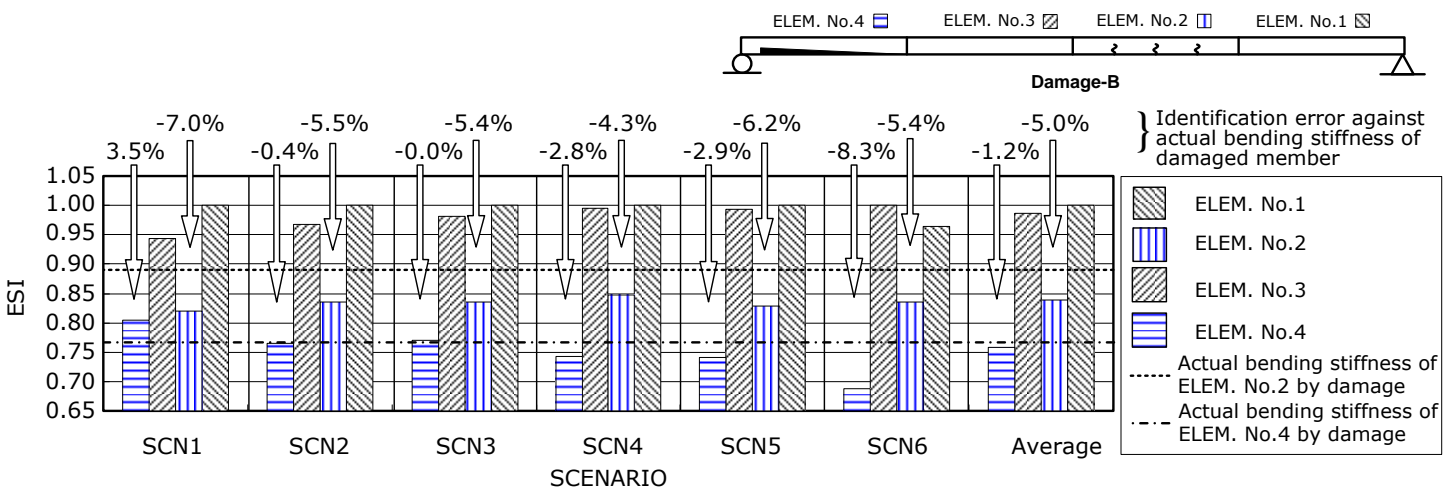

(b)

Fig. 12. Identified damage location and severity by level 3 screening: (a) D1; and (b)

D2. 

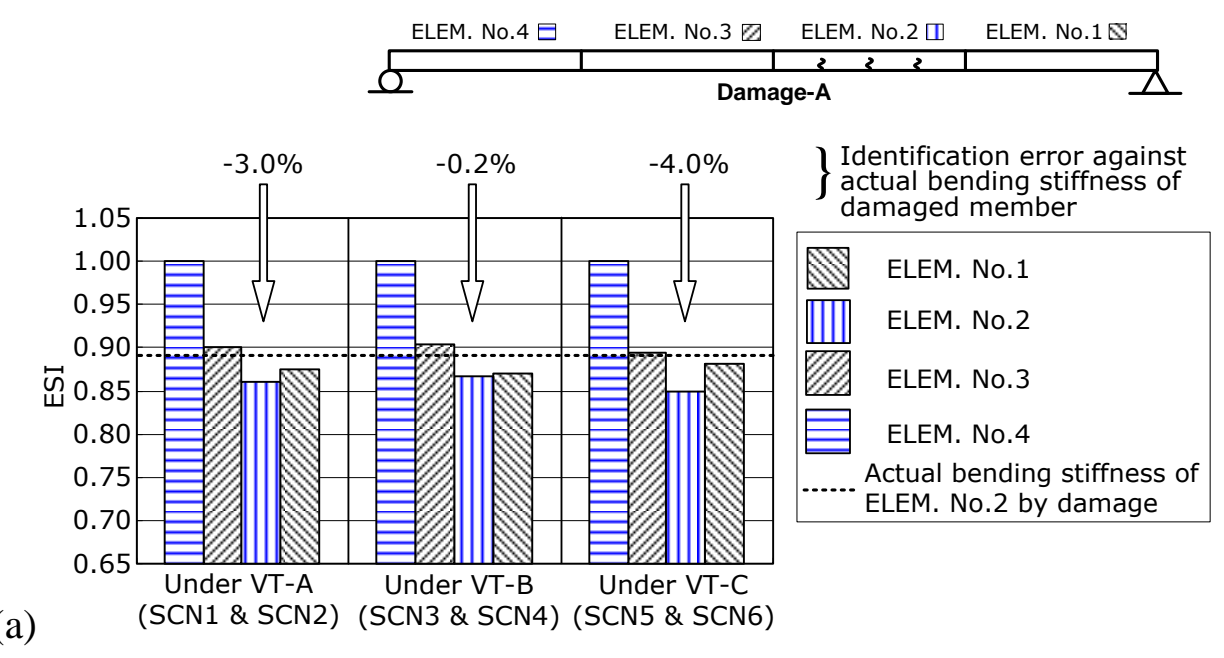

(a)
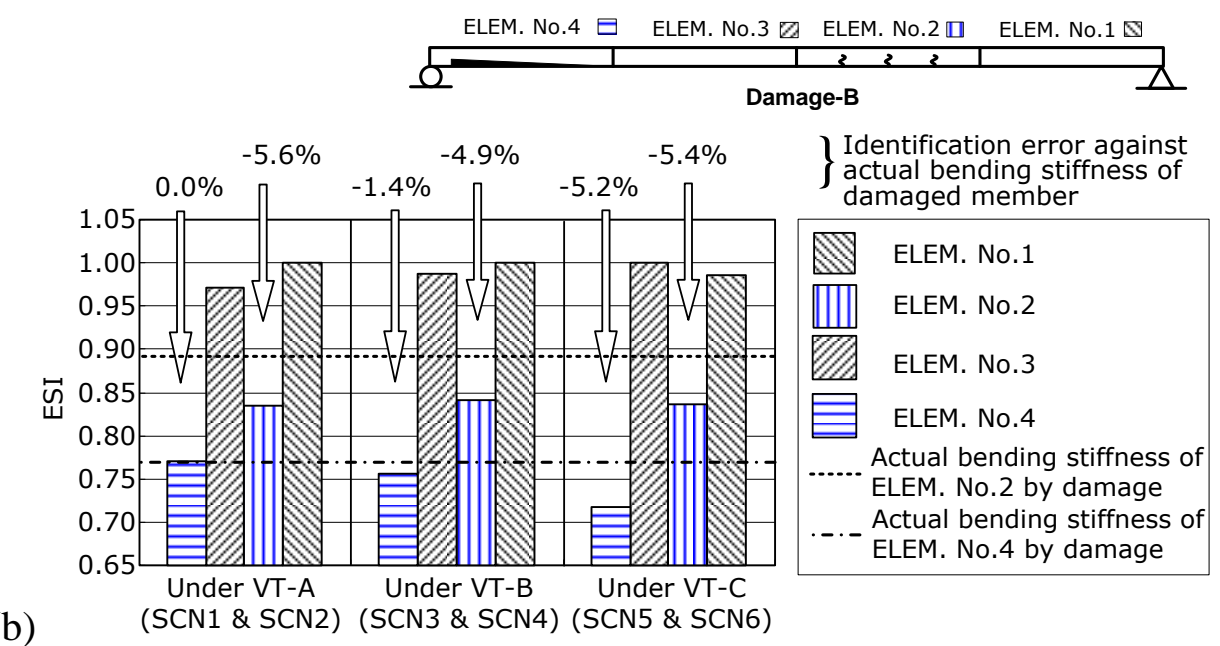

Fig. 13. Identified damage location and severity of the bridge according to vehicle type: (a) with damage at ELEM. No.2 (D1); (b) with damage both at ELEM. No.2 and ELEM. No.4 (D2).

Suspected damage locations and severity of the damage scenario D2 are also well identified in all scenarios as shown in Fig. 12(b). Studying this figure and comparing D1 to D2, it can be seen that the new damage in ELEM. No. 4 can be clearly identified and distinguished from the damage in ELEM. No. 2. The damage severity of each element was identified within the error of $7.0 \%$ for the damaged section I (ELEM. No.2 in Fig. 12(b)) and within the error of $8.3 \%$ for the damaged section II (ELEM. No.4 in Fig. 12(b)).

To examine the effect of vehicle type and speed on the identification result, averaged ESI values are summarized according to the vehicle type and traveling speed as shown in Fig. 13 and Fig. 14 respectively. In those figures, the values of actual ESI 
for the damaged elements, i.e. 0.89 for element No. 2 and 0.77 for element No. 4 , are marked in horizontal virtual lines. The difference between the actual and identified ESIs, referred to as the identification error, is also shown.

For the effect of vehicle types as shown in Fig. 13, the vehicle VT-B, which has the highest frequency for the bounce motion among three vehicles, resulted in the smallest error for identifying severity. However, the identified damage location was obscure, especially for D1. On the other hand, both VT-A and VT-C vehicles, which have smaller frequency for the bounce motion and closer frequency with that of bridge's first bending mode, gave clear damage locations and the identification error rate was less than $5.6 \%$. For D2, reasonable identification for damage severity as well as damage locations was observed without being greatly affected by the vehicle type.
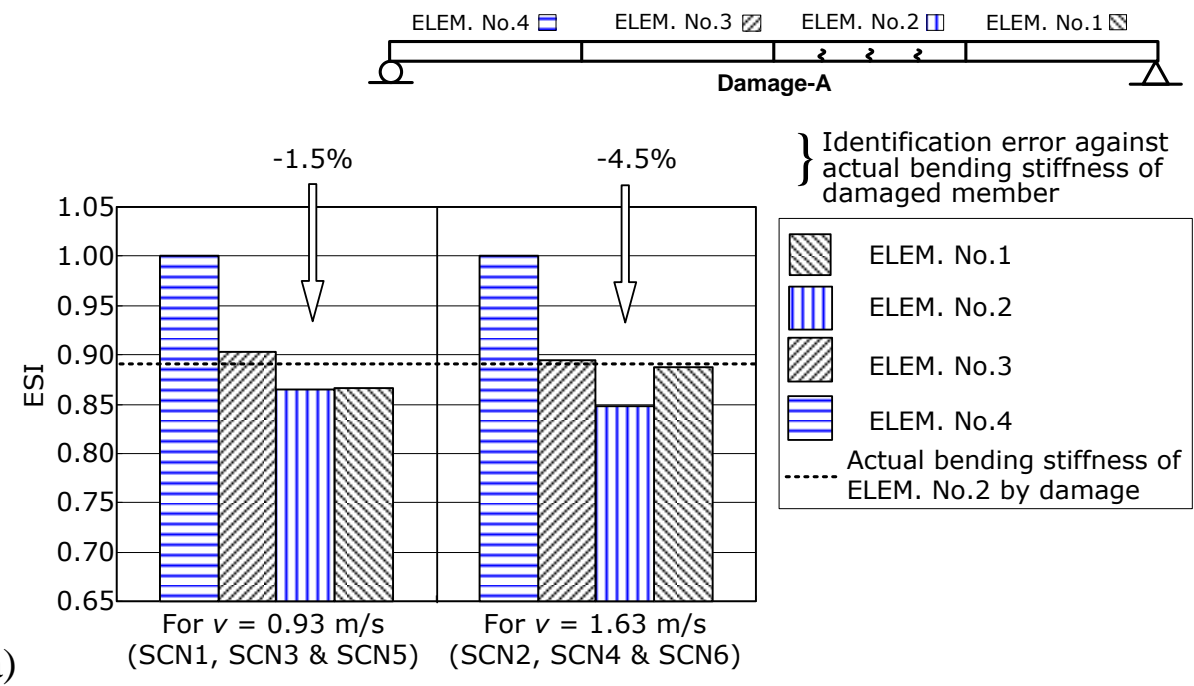

Identification error against J actual bending stiffness of damaged member

ELEM. No.1
ELEM. No.2
ELEM. No.3
ELEM. No.4
Actual bending stiffness of
ELEM. No.2 by damage

(a)
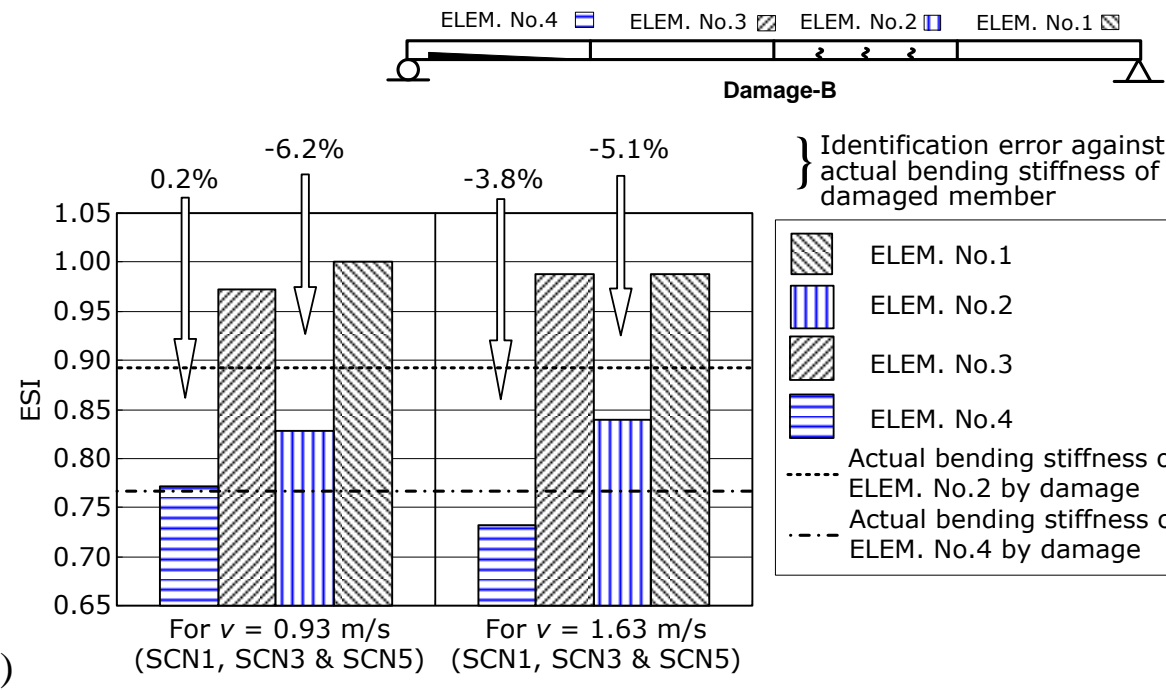

Identification error against actual bending stiffness of damaged member

ELEM. No.1
ELEM. No.2
ELEM. No.3
Actual bending stiffness of
ELEM. No.2 by damage
Actual bending stiffness of
ELEM. No.4 by damage

(b)

Fig. 14. Identified damage location and severity of the bridge according to vehicle speed: (a) with damage at ELEM. No.2 (D1); (b) with damage both at ELEM. No.2 and ELEM. No.4 (D2). 
The effect of the vehicle's travelling speed on identification accuracy is shown in Fig. 14. For D1, the lower travelling speed, $\mathrm{S} 1=0.93 \mathrm{~m} / \mathrm{s}$, gave smaller error for identifying damage severity than that under vehicle speed of $1.63 \mathrm{~m} / \mathrm{s}$. However, the damage location became unclear under the lower speed. For D2, both travelling speeds resulted reasonable identification for damage severity as well as damage locations.

Observations from the experimental investigation demonstrate that the location and severity of damage are both consistently identified without great variation according to vehicle type and speed; even though the vehicle which has similar frequency characteristics with the bridge's fundamental frequency and higher speed may give better chance to identify both severity and location.

\section{Concluding Remarks}

This study investigates feasibility of the drive-by bridge health monitoring through a scaled moving vehicle laboratory experiment. The results can be summarized for each screening level as follows.

Level 1: The bridge frequency was identified in the vehicle spectra. It is clear that selection of vehicle speed is an important factor in the detection of the bridge frequency. The higher speed provides larger magnitude peaks in the spectra but the spectral resolution is not as high as for lower speed, suggesting that in practice, vehicle speed should be selected in order to provide an optimal trade-off between magnitude and resolution. Changes in damping are detected in the vehicle spectra. Observations indicate possibility to detect the bridge frequency and changes in damping from the acceleration measurements of a moving vehicle. However, those approaches are feasible for extracting the natural frequency of bridges within restricted conditions.

Level 2: A clear change of identified dominant frequencies and damping constants is observed despite of their variation, which demonstrates the feasibility of making decisions on the health condition of short span bridges from changes in identified system parameters using the traffic-induced vibration data. However how to quantify and qualify the pattern changes for the structural diagnosis is a remaining task to be studied.

Level 3: The location and severity of damage are constantly identified without great variation according to vehicle type and speed, even though the vehicle with similar frequency characteristics with bridge's fundamental frequency and higher 
speed may give better chance to identify both severity and location. It needs further investigation to adapt for the damage detection even in operational condition.

Throughout the study and for all screening levels, it was clear that lower speed and the vehicle with similar frequency of bounce motion with the fundamental frequency of bridge provides better identification results.

Further investigations are necessary to make the method practically applicable, such as how sensitive the method is under various kinds of damage, even though feasibility of the drive-by bridge inspection was observed through the laboratory experiment. Another great challenge is realizing data acquisition both from moving vehicle and bridge simultaneously.

\section{Acknowledgement}

A part of this study is supported by the Japan Society for the Promotion of Science (Grant-in-Aid for Scientific Research (B) under project No. 24360178), which is gratefully acknowledged.

\section{References}

Adeli, H. and Jiang, X. (2006), "Dynamic fuzzy wavelet neural network model for structural system identification”, J. Struct. Eng., ASCE, 132 (1), 102-111.

Doebling, S.W., Farrar, C.R., Prime, M.B. and Shevitz, D.W. (1996), "Damage identification and health monitoring of structural and mechanical systems from changes in their vibration characteristics: A literature review", Los Alamos National Laboratory Report, LA-3070-MS.

Friswell, M.I. and Mottershead, J.E. (1994), "Finite element model updating in structural dynamics", Kluwer Academic Publishers, 56-77.

Federal Highway Administration (FHWA) (2001). "Phenomenology study of HERMES ground-penetrating radar technology for detection and identification of common bridge deck features", Report FHWA-RD-01-090, U.S. Department of Transportation, June 2001.

Furukawa, T., Fujino, Y., Kubota, K. and Ishii, H. (2007), "Real-time Diagnostic System for Pavements using Dynamic Response of Road Patrol Vehicles (VIMS)", In B. Bakht and A. Mufti (eds), Structural Health Monitoring and Intelligent Infrastructure, CD-ROM. 
Gersch, W., Nielsen, N.N., and Akaike, H. (1973), "Maximum Likelihood Estimation of Structural Parameters from Random Vibration Data", J. Sound Vibr., 31(3), 295-308.

González, A., OBrien, E.J. and McGetrick, P.J. (2010), "Detection of Bridge Dynamic Parameters Using an Instrumented Vehicle", Proceedings of the Fifth World Conference on Structural Control and Monitoring, Tokyo, Japan, paper 34.

Hoshiya, M. and Saito, E. (1984), "Structural identification by extended Kalman filter", J. Eng. Mech., ASCE, 110(12), 1757-1770.

Kim, C.W., Kawatani, M., and Kim, K.B. (2005), "Three-dimensional Dynamic Analysis for Bridge-vehicle Interaction with Roadway Roughness", Comput. Struct., 83, 1627-1645.

Kim, C.W., Kawatani, M., Tsukamoto, M. and Fujita, N. (2008), "Wireless sensor node development for bridge condition assessment", Adv. Sci. Tech., 56, 573-578.

Kim, C.W., and Kawatani M. (2008), "Pseudo-static approach for damage identification of bridges based on coupling vibration with a moving vehicle", Struct. Infrastruct. Eng., 4(5), 371-379.

Kim, C.W. and Kawatani, M. (2009), "Challenge for a Drive-by Bridge Inspection", Proceedings of the 10th International Conference on Structural Safety and Reliability, ICOSSAR2009, Osaka, Japan, 758-765.

Kim, C.W., Kawatani, M., and Hao, J. (2012), "Modal parameter identification of short span bridges under a moving vehicle by means of multivariate AR model", Struct. Infrastruct. Eng., 8(5), 459-472.

Kim, J., Lynch, J.P., Lee, J.J. and Lee, C.G. (2011), "Truck-based mobile wireless sensor networks for the experimental observation of vehicle-bridge interaction", Smart Mater. Struct., 20, doi:10.1088/0964-1726/20/6/065009.

Magalhaes, F. and Cunha, A. (2011), "Explaining operational modal analysis with data from an arch bridge", Mech. Syst. Signal Proc., 25, 1431-1450.

McGetrick, P.J., Gonzalez, A., and OBrien, E.J. (2009), “Theoretical investigation of the use of a moving vehicle to identify bridge dynamic parameters", Insight, 51(8), 433-438.

Nair, K.K., Kiremidjian, A.S. and Law, K.H. (2006), "Time series-based damage detection and localization algorithm with application to the ASCE benchmark structure", J. Sound Vibr., 291, 349-368.

Ni, Y.Q., Zhou, H.F., Chan, K.C., and Ko, J. M. (2008), "Modal flexibility analysis of cable-stayed bridge Ting Kau bridge for damage identification", Comp.-Aided Civ. Inf., 23 (3), 223-236. 
Pappa, R.S., and Ibrahim, S.R. (1981), “A Parametric Study of the Ibrahim Time Domain Modal Identification Algorithm", Shock Vib. Bulletin, No. 51, Part 3, 43-72.

Rizos, P.F., Aspragatos, N., and Dimarogonas, A.D. (1990), "Identification of crack location and magnitude in a cantilever beam from the vibration modes", J. Sound Vibr., 138, 381-388.

Shifrin, E.I. and Ruotolo, R. (1999), "Natural frequencies of a beam with an arbitrary number of cracks", J. Sound Vibr., 222, 409-423.

Shinozuka, M., Yun C.B. and Imai, H. (1982), "Identification of linear structural dynamic systems", J. Eng. Mech. Div., ASCE, 108(6),1371-1390.

Siringoringo, D.M. and Fujino, Y. (2006), "Observed dynamic performance of the Yokohama Bay Bridge from system identification using seismic records", Struct. Control Hlth, 13, 226-244.

Wang, Z. and Fang, T. (1986), "A time-domain method for identifying modal parameters". J. Appl. Mech., ASME, 53(3), 28-32.

Xia, H. and De Roeck, G. (1997), "System identification of mechanical structures by a high-order mulcutivariate autoregressive model", Comput. Struct., 64(1-4), 341-351.

Yang, Y.B., Lin, C.W., and Yau, J.D. (2004), "Extracting Bridge Frequencies from the Dynamic Response of a Passing Vehicle", J. Sound Vibr., 272, 471-493.

Yang, Y.B. and Lin, C.W. (2005), "Vehicle-bridge interaction dynamics and potential applications", J. Sound Vibr., 284, 205-226.

Yang, Y.B. and Chang, K.C. (2009), "Extracting the bridge frequencies indirectly from a passing vehicle: Parametric study", Eng. Struct., 31(10), 2448-2459.

Yin, S.H. and Tang, C.Y. (2011), "Identifying cable tension loss and deck damage in a cable-stayed bridge using a moving vehicle", J. Vib. Acoust., ASME, 133, 021007-1 - 021007-11.

Zhan, J.W., Xia, H., Chen, S.Y. and De Roeck, G. (2011), "Structural damage identification for railway bridges based on train-induced bridge responses and sensitivity analysis", J. Sound Vibr., 330, 757-770. 